\title{
MicroRNA-133b inhibits cell proliferation and promotes apoptosis by targeting cullin 4B in esophageal squamous cell carcinoma
}

\author{
HAIRONG HUANG $^{1 *}$, YANG XU ${ }^{1 *}$, ZHONG GUO $^{1}, \mathrm{XI} \mathrm{CHEN}^{1}$, SHAIGUANG JI $^{1}$ and ZHIYUN XU ${ }^{2}$ \\ ${ }^{1}$ Department of Cardiothoracic Surgery, Jinling Hospital, Medical School of Nanjing University, Nanjing, Jiangsu 210002; \\ ${ }^{2}$ Department of Cardiothoracic Surgery, Changhai Hospital, Second Military Medical University, Shanghai 200433, P.R. China
}

Received May 21, 2017; Accepted December 8, 2017

DOI: $10.3892 /$ etm.2018.5906

\begin{abstract}
RNAs (miRs) serve a role as modulators during carcinogenesis. It has been demonstrated that the expression of miR-133b is decreased in a variety of tumor tissues and cell lines and serves a suppressive role in the proliferation and apoptosis of different types of tumor cells. However, its effect on esophageal squamous cell carcinoma (ESCC) cells remains unclear. In the current study, the expression of mature miR-133b was measured using reverse transcription-quantitative polymerase chain reaction and the results indicated that miR-133b was significantly downregulated in ESCC tissues and various ESCC cell lines. The overexpression of miR-133b significantly inhibited the proliferation and promoted the apoptosis of KYSE150 and Eca-109 cells. Furthermore, it was demonstrated that cullin 4B (CUL4B) promotes ESCC cell proliferation and inhibits apoptosis by activating the protein kinase $\mathrm{B} /$ glycogen synthase kinase $3 \beta / \beta$-catenin pathway. Taken together, these results demonstrate that miR-133b/CUL4B serves a tumor suppressive role during ESCC progression and may therefore be used as a potential target to treat patients with ESCC.
\end{abstract}

\section{Introduction}

Esophageal cancer is the eighth most common cancer worldwide, with $\sim 480,000$ new cases and 400,000 associated mortalities per year (1). Histologically, there are two main forms of esophageal cancer: Esophageal adenocarcinoma and esophageal squamous cell carcinoma (ESCC), which represents $90 \%$ of all esophageal cancer cases (2). Currently, surgery is the only way to treat patients with esophageal cancer and the overall 5 -year survival rate is $14-18 \%$. This is due to the fact that surgery can only be performed in a limited number of

Correspondence to: Dr Zhiyun Xu, Department of Cardiothoracic Surgery, Changhai Hospital, Second Military Medical University, 168 Changhai Road, Shanghai 200433, P.R. China

E-mail: xzy_changhai@163.com

${ }^{*}$ Contributed equally

Key words: microRNA-133b, esophageal squamous cell carcinoma, proliferation, apoptosis, cullin 4B patients, as in many cases tumors are inoperable (3). Although genetic and epigenetic alterations underpin the development of ESCC, the molecular mechanisms underlying neoplastic progression remain unclear $(4,5)$. Therefore, improving understanding of the molecular biology of ESCC is critical to facilitate the development of more effective diagnostic and therapeutic strategies for ESCC.

MicroRNAs (miRs) are small non-coding RNAs 22 nucleotides long that bind to the 3'-untranslated region (3'-UTR) of target mRNA (6). Previous studies have demonstrated that different types of miRs regulate the proliferation, apoptosis, invasion, metastasis and the epithelial-mesenchymal transition of tumor cells $(7,8)$. The aberrant expression of miRs has also been identified in ESCC $(9,10)$. The upregulated expression of miR-21 may promote cell proliferation, migration and resistance to apoptosis via the phosphatase and tension homolog/phosphoinositide 3 kinase/protein kinase b signaling pathway in ESCC (11). miR-let-7 is downregulated in ESCC and is considered to be a tumor suppressor, as it regulates the interleukin-6/signal transducer and activator of transcription 3 signaling pathway during neoplastic progression (12). Additionally, it has been demonstrated that miR-142-3p expression is associated with histological differentiation and may be a potential independent prognostic factor in patients with ESCC following surgery (13). It has been demonstrated that miR-133b decreases the invasiveness of esophageal cancer by inhibiting the expression of fascin actin-bundling protein 1 (FSCN1) (14). However, the molecular mechanisms underlying the function of miR-133b in proliferation and apoptosis of esophageal cancer cells remains unknown.

The results of the current study demonstrated that miR-133b expression was downregulated in ESCC tissues and cell lines and its low expression was associated with the clinicopathological features of patients with ESCC. Overexpression of miR-133b in ESCC cell lines decreased cell proliferation and promoted cell apoptosis. Cullin 4B (CUL4B) has been identified as a direct target of miR-133b and it was demonstrated that miR-133b functions as a tumor suppressor by negatively regulating CUL4B expression. Furthermore, it was demonstrated that CUL4B promotes ESCC cell proliferation and inhibits apoptosis by activating the protein kinase $\mathrm{B} / \mathrm{glycogen}$ synthase kinase $3 \beta / \beta$-catenin (AKT/GSK3 $\beta / \beta$-catenin) pathway. Thus, the results of the present study suggest that miR-133b/CUL4B may be a promising therapeutic target for ESCC. 


\section{Materials and methods}

Tissue collection. Primary ESCC tissues and adjacent non-tumor tissues samples ( $>5 \mathrm{~cm}$ from the edge of tumor) were obtained from 47 untreated patients ( 27 males and 20 females; mean age of 57 years) undergoing primary surgical resection at the Department of Cardiothoracic Surgery, Jinling Hospital (Nanjing, China) between January 2014 and August 2015. The clinical staging of tumors was performed using the seventh edition of the American Joint Committee on Cancer Staging Manual (15). Samples were snap-frozen in liquid nitrogen prior to RNA isolation and reverse transcription-quantitative polymerase chain reaction (RT-qPCR). The current study was approved by the Human Ethics Committee of Jinling Hospital and written informed consent was obtained from each study participant.

Cell lines and cell culture. Six human ESCC cell lines (TE-1, TE-8, KYSE150, KYSE450, Eca-109 and EC9706) and the normal human esophageal epithelial cell line HEECs (cat. no. BNCC337729) were purchased from the Shanghai Institute of the Chinese Academy of Sciences (Shanghai, China). Another normal human esophageal epithelial cell line Het-1A was purchased from American Type Culture Collection (Manassas, VA, USA). TE-1, TE-8, Eca-109, EC9706 and Het-1A cells were cultured in Roswell Park Memorial Institute medium-1640 (Thermo Fisher Scientific Inc., Waltham, MA, USA), and KYSE150, KYSE450 and HEECs cells were cultured in Dulbecco's modified Eagle's medium (Thermo Fisher Scientific, Inc.). These media were supplemented with $10 \%$ fetal bovine serum (Invitrogen; Thermo Fisher Scientific, Inc.), $100 \mathrm{U} / \mathrm{ml}$ penicillin and $100 \mu \mathrm{g} / \mathrm{ml}$ streptomycin in a humidified atmosphere at $37^{\circ} \mathrm{C}$ with $5 \% \mathrm{CO}_{2}$.

$R T-q P C R$. Tissues samples and culture cells were treated with TRIzol ${ }^{\circledR}$ reagent (Invitrogen; Thermo Fisher Scientific, Inc.) for total RNA extraction, following the manufacturer's protocols. For miR-133b quantification, 100 ng total RNA was reverse transcribed using the specific stem-loop RT primer and the TaqMan MicroRNA Reverse Transcription kit (Applied Biosystems; Thermo Fisher Scientific, Inc.) was used. The cDNA were quantified using a TaqMan MicroRNA assay (Applied Biosystems; Thermo Fisher Scientific, Inc.) and normalized to U6 RNA levels. The qPCR reaction conditions were as follows: Initial denaturation at $95^{\circ} \mathrm{C}$ for $10 \mathrm{~min}$ and subsequently 40 cycles of $95^{\circ} \mathrm{C}$ for $15 \mathrm{sec}$ and $60^{\circ} \mathrm{C}$ for $1 \mathrm{~min}$. For CUL4B mRNA analysis, cDNA was synthesized from $1 \mu \mathrm{g}$ total RNA using PrimeScript ${ }^{\mathrm{TM}} \mathrm{RT}$ reagent kit (Takara Biotechnology Co., Ltd., Dalian, China). qPCR was performed using a TaqMan RT-qPCR assay (Applied Biosystems; Thermo Fisher Scientific, Inc.) and normalized to GAPDH mRNA levels. The qPCR reaction conditions were as follows: Initial denaturation at $95^{\circ} \mathrm{C}$ for $5 \mathrm{~min}$ and subsequently 40 cycles of $95^{\circ} \mathrm{C}$ for $15 \mathrm{sec}, 60^{\circ} \mathrm{C}$ for $15 \mathrm{sec}$ and $70^{\circ} \mathrm{C}$ for $20 \mathrm{sec}$. Each sample was analyzed in triplicate using an ABI 7500 Fast Real-Time PCR system (Applied Biosystems; Thermo Fisher Scientific, Inc.). Relative expression levels were evaluated using the $2^{-\triangle \Delta C q}$ method (16). The following primers were used for qPCR: miR-133b forward, 5'-TTTGGTCCCCTTCAA CCAGCTA-3' and reverse, 5'-GTGCAGGGTCCGAGGT-3'; and CUL4B forward, 5'-GGGAAAGGAATGGTGAA-3' and reverse, 5'-TGCATAGAGCCGGTTAG-3'. The primers used for the internal controls were as follows: U6 forward, 5'-CTC GCTTCGGCAGCACA-3' and reverse, 5'-AACGCTTCACGA ATTTGCGT-3'; and GAPDH forward, 5'-TGACTTCAACAG CGACACCCA-3' and reverse, 5'-CACCCTGTTGCTGTA GCCAAA-3'.

miR-133b transfection. The mature miR-133b sequence (5'-UUUGGUCCCCUUCAACCAGCUA-3') was obtained from the miRBase database (http://www.mirbase.org/). Subsequently, $0.5 \mu \mathrm{g}$ miR-133b cDNA sequence was synthesized and inserted into the pcDNA3.1 vector to generate a human pcDNA-miR-133b plasmid. For transfection, KYSE150 or Eca-109 cells ( $\sim 5 \times 10^{5} /$ well) were seeded in 6 -well plates. After $24 \mathrm{~h}$, the cells were transfected with $3 \mu \mathrm{g}$ pcDNA-miR-NC control vector or pcDNA-miR-133b plasmids using Lipofectamine ${ }^{\mathrm{TM}} 2000$ (Invitrogen; Thermo Fisher Scientific, Inc.). Cultures were incubated for $72 \mathrm{~h}$ prior to collecting samples for subsequent western blotting and RT-qPCR analysis.

Cell proliferation assay. Cell proliferation was determined by culturing $\sim 5 \times 10^{3}$ cells/well on 96-well plates and cells were transiently transfected with $2 \mu \mathrm{g}$ pcDNA-miR-133b/miR-NC or pcDNA-CUL4B/empty plasmids (FulenGen, Guangzhou, China) using Lipofectamine ${ }^{\mathrm{TM}}$ 2000. After $72 \mathrm{~h}, 10 \mu \mathrm{l}$ Cell Counting kit-8 (CCK-8) solution (Dojindo Molecular Technologies, Inc., Kumamoto, Japan) was added to each well and cells were incubated for $1 \mathrm{~h}$ at $37^{\circ} \mathrm{C}$. Optical density was then detected at a wavelength of $450 \mathrm{~nm}$ using an Epoch Microplate Spectrophotometer (BioTek Instruments, Inc., Winooski, VT, USA). Each sample was analyzed in triplicate and data analysis was performed using the mean of the results.

Cell apoptosis assay. Cell apoptosis was analyzed using an Annexin V-fluorescein isothiocyanate (FITC) Apoptosis Detection kit (Beyotime Institute of Biotechnology, Haimen, China). Trypsinized cells were washed three times with PBS and stained with FITC-labeled anti-Annexin V antibody and propidium iodide in the dark for $10 \mathrm{~min}$ at room temperature. Cells were then analyzed using the BD FACSCalibur flow cytometer (BD Biosciences, Franklin Lakes, NJ, USA) and FlowJo 7.6.1 software (FlowJo LLC, Ashland, OR, USA).

Western blot analysis. Transfected cells were washed once in PBS and lysed in radioimmunoprecipitation lysis buffer (Beyotime Institute of Biotechnology, Jiangsu, China) and protein concentration was determined using a BCA protein assay kit. A total of $50 \mu \mathrm{g} /$ lane protein was separated by $10 \%$ SDS-polyacrylamide gel electrophoresis and transferred onto $0.22 \mu \mathrm{m}$ polyvinylidene difluoride membranes, which was incubated with 5\% fat-free skimmed milk in Tris-buffered saline containing $0.05 \%$ Tween-20 for $1 \mathrm{~h}$ at room temperature. Membranes were incubated overnight at $4^{\circ} \mathrm{C}$ with rabbit anti-CUL4B (cat. no. ab157103; 1:1,000; Abcam, Cambridge, UK), $\beta$-catenin (cat. no. ab32572; 1:1,000; Abcam), GSK-3 $\beta$ (cat. no. 9315; 1:1,000; Cell Signaling Technology, Danvars, MA, USA), phosphorylated (p)-GSK-3 $\beta^{\text {Tyr216 }}$ (cat. no. ab75745; 1:1,000; Abcam) and $\beta$-actin (cat. no. sc-130656; 1:1,000; Santa 
Cruz Biotechnology, Inc., Dallas, TX, USA) primary antibodies. Following extensive washing with PBS supplemented with $0.1 \%$ Triton $\mathrm{X}-100$, membranes were incubated with horseradish peroxidase-conjugated goat anti-rabbit secondary antibody (cat. no. ab205718; 1:1,500; Abcam) for $1 \mathrm{~h}$ at room temperature. Images were visualized using an enhanced chemiluminescent system (EMD Millipore, Billerica, MA, USA). Relative band intensities were determined by densitometry using Quantity One 4.6.2 software (Bio-Rad Laboratories, Inc., Hercules, CA, USA).

Luciferase reporter assays. The 3'-UTR of CUL4B with wild-type or mutant binding sites for miR-133b was amplified and subcloned into the pGL3 vector (Promega Corp., Madison, WI, USA), respectively. Eca-109 and KYSE150 cells were co-transfected with $150 \mathrm{ng}$ miR-133b or miR-negative control plasmids and $50 \mathrm{ng}$ pGL3-CUL4B wild-type or mutant reporter plasmids using Lipofectamine 2000. A total of $48 \mathrm{~h}$ following transfection, the Dual-luciferase assay kit (Promega, Madison, WI, USA) was used to determine the luciferase activity. Renilla luciferase activity was normalized to the Firefly luciferase activity. Each experiment was performed in triplicate.

Bioinformatic analysis. The target gene of miR-133b was identified and compared using the online target prediction algorithms miRanda (http://www.microrna.org/), miRWalk (http://www. umm.uni-heidelberg.de/apps/zmf/mirwalk/predictedmirnagene. html) and PicTar (http://www.pictar.org/).

Statistical analysis. All statistical analyses were performed using SPSS software 15.0 (SPSS, Inc., Chicago, IL, USA). Data are expressed as the mean \pm standard error of the mean from $\geq 3$ independent experiments. Statistical significance was evaluated using a Student's t-test (two-tailed), one-way analysis of variance followed by a Tukey's post hoc test and the Mann-Whitney test. Pearson's correlation analysis was used to measure the correlation between CUL4B and miR-133b expression. $\mathrm{P}<0.05$ was determined to indicate a statistically significant difference.

\section{Results}

miR-133b expression is downregulated in human ESCC tissues and cell lines. The biological function of miR-133b in the pathogenesis of ESCC was investigated by examining miR-133b expression in 47 paired ESCC and adjacent non-tumor tissues by RT-qPCR. miR-133b expression was significantly downregulated in ESCC samples compared with adjacent normal tissues ( $\mathrm{P}<0.01$; Fig. 1A). Similarly, miR-133b expression in the six ESCC cell lines TE-1 $(\mathrm{P}<0.01)$, TE-8 $(\mathrm{P}<0.05)$, KYSE150 $(\mathrm{P}<0.05)$, KYSE450 $(\mathrm{P}<0.05)$, Eca-109 $(\mathrm{P}<0.01)$, and EC9706 $(\mathrm{P}<0.05)$, was significantly lower than that in the normal esophageal epithelial cell lines Het-1A and HEEC (Fig. 1B). miR-133b expression was also associated with tumor stage $(\mathrm{P}<0.05)$, tumor size $(\mathrm{P}<0.05)$ and differentiation status $(\mathrm{P}<0.05)$; however, it was not associated with other clinicopathological factors of patients with ESCC (Table I).

CUL4B is a correlated target gene of miR-133b in ESCC cells. The target gene of miR-133b in ESCC was identified using the online target prediction algorithms. Based on the Gene Expression Omnibus database analysis (17), it was revealed that human CUL4B, an important ubiquitilation molecule associated with apoptosis, contained the conserved putative miR-133b target site (Fig. 2A). Dual-luciferase reporter analysis indicated that co-expression of miR-133b significantly inhibited luciferase activity in KYSE150 $(\mathrm{P}<0.01)$ and Eca-109 $(\mathrm{P}<0.05)$ cells containing the CUL4B-3'-UTR reporter plasmid, compared with those containing the mutant plasmid (Fig. 2B and C). Endogenous expression of CUL4B was significantly inhibited following transfection of miR-133b in KYSE150 and Eca-109 cells compared with the control ( $\mathrm{P}<0.05$; Fig. 2D). Furthermore, CUL4B expression was significantly upregulated in ESCC tissues compared with adjacent non-tumor tissues $(\mathrm{P}<0.05$; Fig. $2 \mathrm{E})$ and there was a negative correlation between miR-133b and CUL4B mRNA levels in ESCC tissues (Fig. 2F). These results indicate that CUL4B is a direct target of miR-133b and suggest that miR-133b may exert its effect by inhibiting CUL4B expression.

miR-133b inhibits cell proliferation and promotes apoptosis by targeting CUL $4 B$ in ESCC. The biological function of miR-133b/CUL4B in ESCC was analyzed by transfecting miR-133b and/or CUL4B into KYSE150 and Eca-109 cells (Fig. 3A). The role of miR-133b/CUL4B on the proliferation of ESCC cells was investigated using a CCK-8 assay and the results indicated that overexpression of $\mathrm{miR}-133 \mathrm{~b}$ significantly decreased the proliferation of KYSE150 and Eca-109 cells compared with cells transfected with the negative control $(\mathrm{P}<0.05$; Fig. 3B). However, reintroduction of CUL4B into miR-133b-transfected KYSE150 or Eca-109 cells significantly reversed the effects of miR-133b on ESCCC cell proliferation $(\mathrm{P}<0.05$; Fig. 3B). Additionally, overexpression of miR-133b significantly increased apoptosis in KYSE150 cells, which was significantly reversed when CUL4B was reintroduced into miR-133b-transfected KYSE150 cells (all $\mathrm{P}<0.05$; Fig. 3C and D). In Eca-109 cells, miR-133b overexpression also significantly increased levels of apoptosis and the reintroduction of CUL4B inhibited the effects of apoptosis $(\mathrm{P}<0.05$; Fig. 3E and F).

miR-133b inhibits the AKT/GSK3 $\beta / \beta$-catenin pathway by downregulating $C U L 4 B$ in ESCC. It has been demonstrated that CUL4B activates AKT/GSK3 $\beta / \beta$-catenin signaling and may promote proliferation and invasion in malignant neoplasms (18). Therefore, it was hypothesized that miR-133b/CUL4B inhibits ESCC cell proliferation and promotes apoptosis by inhibiting AKT/GSK3 $\beta / \beta$-catenin signaling pathway. After transfection of miR-133b or miR-NC plasmid, CUL4B, GSK-3 $\beta$, p-GSK $3 \beta^{\text {Tyr216 }}$ and $\beta$-catenin levels were measured using western blot analysis. The results indicated that the expression of GSK3 $\beta(\mathrm{P}<0.01)$ and $\mathrm{p}-\mathrm{GSK} 3 \beta(\mathrm{P}<0.05)$ were significantly increased in KYSE150 cells transfected with miR-133b compared with those transfected with the negative control (Fig. 4A and B). By contrast, the expression of the CUL4B $(\mathrm{P}<0.01)$ and downstream proliferation/apoptosis-associated target protein $\beta$-catenin $(\mathrm{P}<0.05)$ were significantly decreased. Similarly, levels of GSK $3 \beta$ and p-GSK3 $\beta$ were significantly increased $(\mathrm{P}<0.05)$, but the levels of CUL4B and $\beta$-catenin were 
Table I. Association between miR-133b expression and clinicopathological features of patients with ESCC.

miR-133b expression

Characteristics

Patients $(n=47)$

High $(n=24)$

Low (n=23)

P-value

Age (years)

$>60$

29

15

14

0.417

$<60$

18

8

10

Sex

Male

Female

Tumor localization

Upper third 12

Middle third

Lower third

Tumor stage

I+II

25

19

11

7

9

14

Tumor size $(\mathrm{cm})$

$<5$ $\geq 5$

Positive
28

19

\section{8}

12

20

7

19

(2)

6

15

9

\section{8}

16

8

$0.041^{\mathrm{a}}$

$0.034^{\mathrm{a}}$

17

${ }^{a} \mathrm{P}<0.05$. Statistical significance was evaluated using Pearson's $\chi^{2}$ test. miR-133b, microRNA-133b; ESCC, esophageal squamous cell carcinoma.
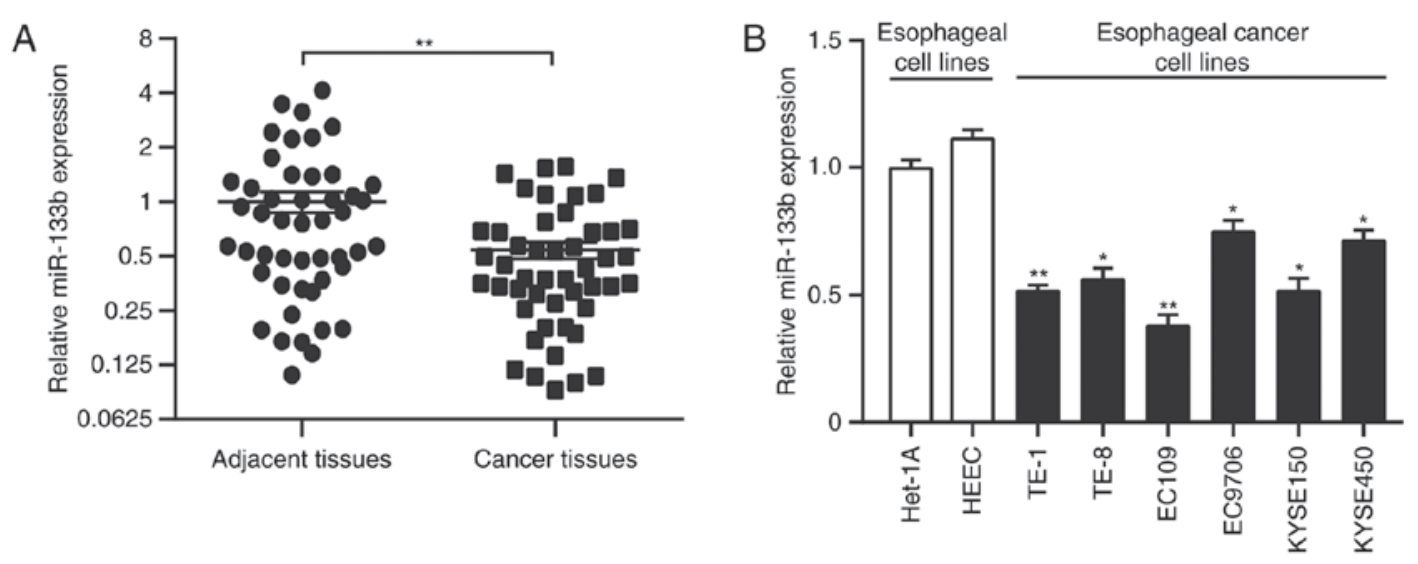

Figure 1. miR-133b expression in ESCC tissues and cell lines. (A) miR-133b expression was downregulated in ESCC tissues compared with the corresponding non-tumorous tissues. (B) miR-133b expression was downregulated in the six ESCC cell lines compared with the two normal esophageal epithelial cell lines. Data were normalized to GAPDH expression. Data are expressed as the mean \pm standard error of the mean. Each sample was analyzed in triplicate. ${ }^{\mathrm{P}}<0.05$, ${ }^{* *} \mathrm{P}<0.01$. miR-133b, microRNA-133b; ESCC, esophageal squamous cell carcinoma.

significantly decreased $(\mathrm{P}<0.05)$ in Eca-109 cells transfected with miR-133b compared with cells transfected with negative control (Fig. 4C and D). Taken together, these results indicate that miR-133b/CUL4B may affect ESCC cell proliferation and apoptosis by regulating the $\mathrm{AKT} / \mathrm{GSK} 3 \beta / \beta$-catenin pathway.

\section{Discussion}

miR-133b is a muscle-specific molecular marker, which serves a role in skeletal muscle development, myoblast differentiation and myogenic-associated disease $(19,20)$. It has been demonstrated that miR-133b serves a suppressive role during tumor 
A

Position of CUL4B 3'UTR

CUL4B 3'UTR-wT UUUUUUACUACCGAUGACCAAA

hsa-miR-133b

CUL4B 3'UTR-mut

AUCGACCAACUUCCCO

(1)

UUUUUUACUACCGAUGCCCGAA
B

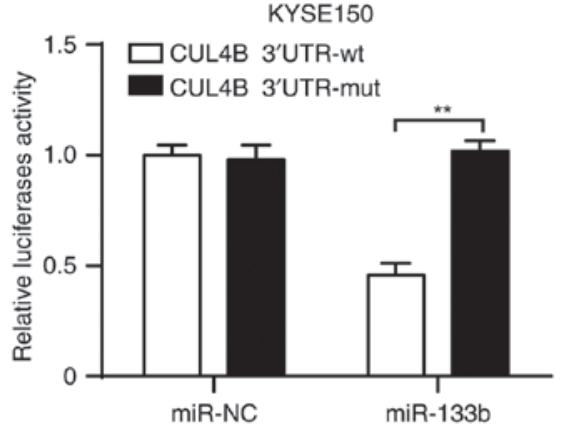

C
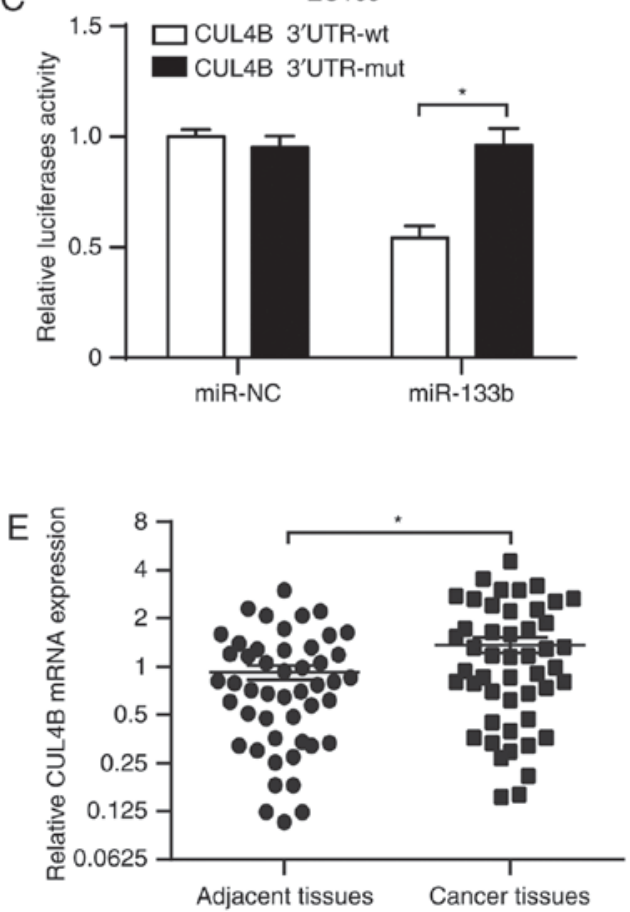

D
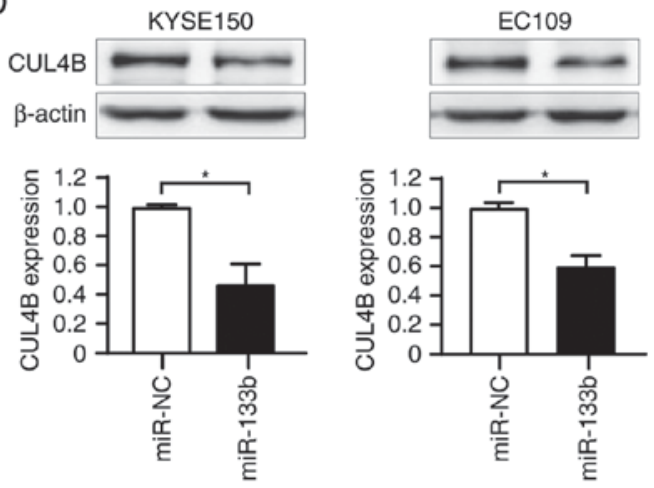

$\mathrm{F}$

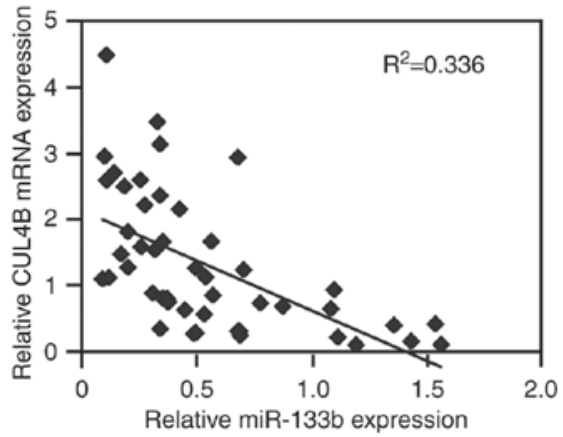

Figure 2. CUL4B is a target gene of miR-133b. (A) Sequence alignment of miR-133b and its conserved target site in the CUL4B-3'-UTR. (B) Relative luciferase activity in KYSE150 cells co-transfected with pGL3-CUL4B wt or mut reporter plasmids containing miR-133b or niR-NC. (C) Relative luciferase activity in Eca-109 cells co-transfected with pGL3-CUL4B wt or mut reporter plasmids containing miR-133b or niR-NC. (D) Endogenous expression of CUL4B was significantly inhibited by transfection of miR-133b in KYSE150 and Eca-109 cells. (E) CUL4B mRNA expression was upregulated in ESCC tissues compared with the corresponding non-tumorous tissues as evaluated by reverse transcription-quantitative polymerase chain reaction. (F) The expression of miR-133b was negatively correlated with the expression of CUL4B in ESCC tissues. Data are expressed as the mean \pm standard error of the mean. Each sample was analyzed in triplicate. ${ }^{*} \mathrm{P}<0.05,{ }^{* *} \mathrm{P}<0.01$. CULB4B, cullin 4B; miR-133b, microRNA-133b; ESCC, esophageal squamous cell carcinoma; miR-NC, microRNA negative control; 3'-UTR, 3'-untranslated region; wt, wild-type; mut, mutant.

growth, invasion, metastasis and apoptosis. miR-133b inhibits gastric cancer cell metastasis in vitro and in vivo by directly suppressing the expression of zinc finger protein Gli1 (21). miR-133b promotes the apoptosis and inhibits the proliferation of osteosarcoma cells by directly targeting B cell lymphoma-2 like protein (22). Downregulation of miR-133b in colorectal cancer tissues compared with adjacent non-tumorous tissues is associated with the poor survival of patients (23). Furthermore, it has been demonstrated that miR-133b inhibits the invasiveness of esophageal types of cancer by inhibiting FSCN1 expression (14). However, the molecular mechanisms of miR-133b in ESCC cell apoptosis and proliferation remain unknown.

The results of the present study demonstrated that miR-133b significantly decreased tumor cell proliferation and promoted apoptosis in vitro. These results indicate that miR-133b may be used as a novel method of treating patients with ESCC.
miR-133b levels were also highly associated with tumor stage and differentiation status; differentiation-associated miR-133b levels may be used to predict tumor progression in patients with ESCC that have undergone surgery; however, further studies are required to validate this.

It was also demonstrated that miR-133b/CUL4B served a role in ESCC cell growth and apoptosis. CUL4B is a member of the cullin family and forms a complex that functions as an E3 ubiquitin ligase and catalyzes the polyubiquitination of specific protein substrates in the cell $(24,25)$. Previous studies have demonstrated that CUL4B expression is significantly upregulated in various types of human cancer, promoting cell proliferation, invasion and tumorigenesis (26-28). For example, CUL4B promotes the proliferation and inhibits the apoptosis of osteosarcoma and glioblastoma cells $(26,27)$. In addition, CUL4B is a novel prognostic marker correlating with colon cancer pathogenesis and progression (28). The mechanism 

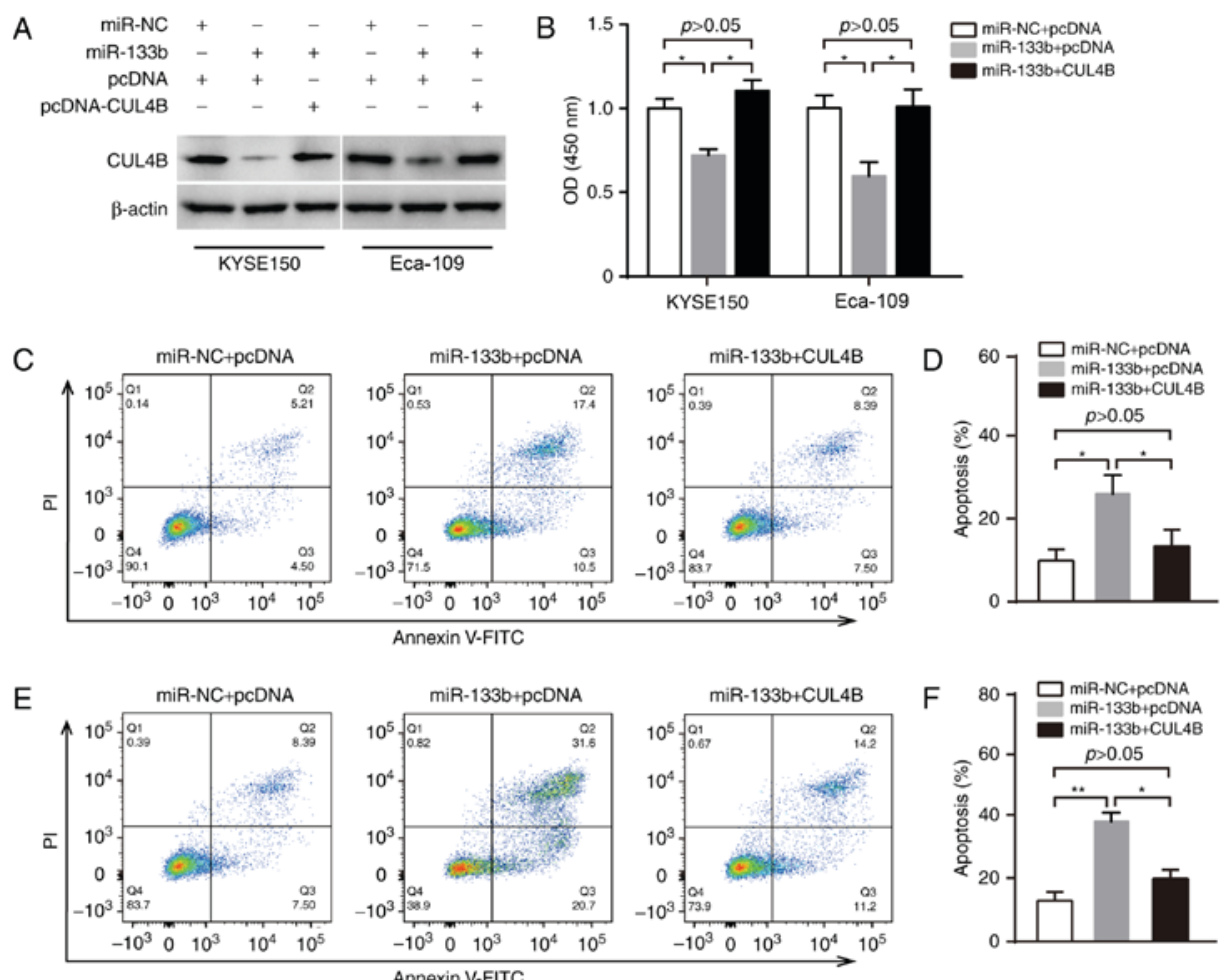

Figure 3. miR-133b inhibits cell proliferation and promotes apoptosis by targeting CUL4B in ESCC. (A) KYSE150 and Eca-109 cells were co-transfected with miR-133b/miR-NC and CUL4B/empty vectors for $72 \mathrm{~h}$ and the expression of CUL4B was evaluated by western blotting. (B) Overexpression of miR-133b inhibited the proliferation of ESCC cells and these effects on cell proliferation were significantly reversed by transfection with CUL4B. (C) Flow cytometric analysis of apoptosis in KYSE150 cells. (D) Overexpression of miR-133b in KYSE150 cells significantly increased cell apoptosis and these effects on apoptosis were significantly reversed by transfection with CUL4B. (E) Flow cytometric analysis of apoptosis in Eca-109 cells. (F) Overexpression of miR-133b in Eca-109 cells increased cell apoptosis and the effects of apoptosis were significantly reversed by transfection with CUL4B. Data are expressed as the mean \pm standard error of the mean. Each sample was analyzed in triplicate. ${ }^{*} \mathrm{P}<0.05,{ }^{* *} \mathrm{P}<0.01$. CULB4B, cullin 4B; miR-133b, microRNA-133b; miR-NC, microRNA negative control; ESCC, esophageal squamous cell carcinoma; PI, propidium iodide; FITC, fluorescein isothiocyanate.
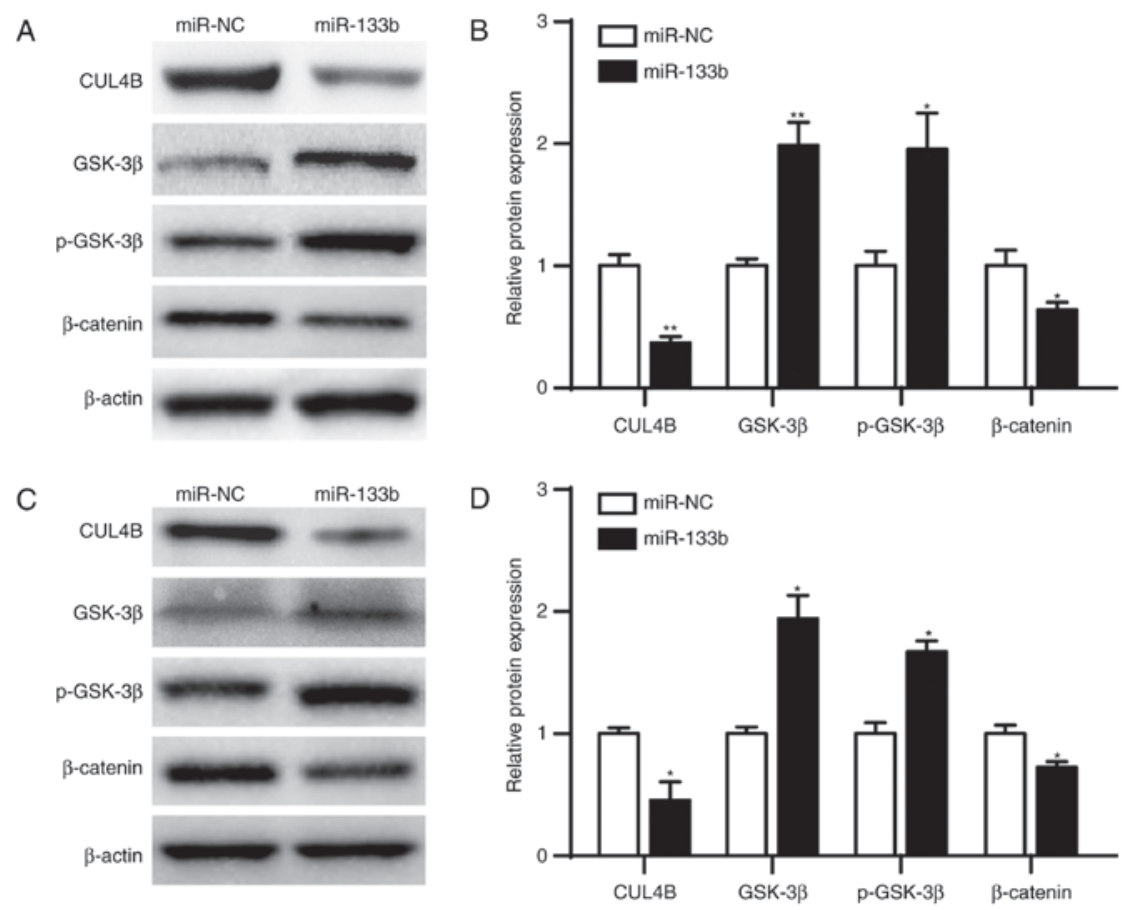

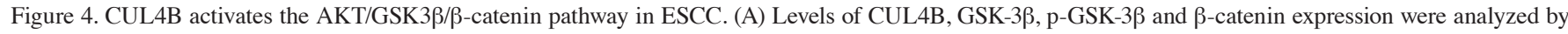
western blot analysis in KYSE150 cells transfected with miR-NC or miR-133b. (B) Quantification of western blot analysis. (C) Levels of CUL4B, GSK-3 $\beta$, p-GSK-3 $\beta$ and $\beta$-catenin expression were analyzed by western blot analysis in Eca-109 cells transfected with mi-NC or miR-133b. (D) Quantification of western blot analysis Data are expressed as the mean \pm standard error of the mean. Each sample was analyzed in triplicate. ${ }^{*} \mathrm{P}<0.05,{ }^{* * *} \mathrm{P}<0.01$ vs. miR-NC. CULB4B, cullin 4B; miR-133b, microRNA-133b; miR-NC, microRNA negative control; ESCC, esophageal squamous cell carcinoma; Akt, protein kinase b; p-, phosphorylated; GSK-3 $\beta$, glycogen synthase kinase $3 \beta ; \mathrm{NC}$, negative control. 
underlying CUL4B function in cancer progression remains unclear; however, CUL4B may serve a role in epigenetic changes, including heterochromatin formation, histone modification, parental imprinting or X-chromosome inactivation $(29,30)$. CUL4B also promotes cell cycle progression and tumorigenesis via degradation of numerous cyclin-dependent kinase inhibitors or p53 protein $(31,32)$. In addition, CUL4B serves an important role in stabilizing $\beta$-catenin against proteasomal degradation in multiple signaling pathways. The results of the current study indicate that CUL4B activates the AKT/GSK3 $\beta / \beta$-catenin pathway and may affect ESCC cell proliferation and apoptosis by regulating this pathway. These results provide important insights into the CUL4B pathway and shed light on the functional importance of proliferation and apoptosis in ESCC.

In conclusion, the current study demonstrated that miR-133b is downregulated in ESCC, and its expression is associated with advanced tumor stage and the differentiation status of patients with ESCC. Additionally, it was determined that miR-133b serves a crucial role in inducing proliferation and apoptosis by directly targeting CUL4B and may therefore be a novel therapeutic target to treat patients with ESCC.

\section{Acknowledgements}

Not applicable.

\section{Funding}

No funding was received.

\section{Availability of data and materials}

All data generated or analyzed during this study are included in this published article.

\section{Authors' contribution}

HH designed methods and experiments, performed the laboratory experiments and analyzed the data. YX and ZG co-designed the cell proliferation and apoptosis experiments and collaborated on associated data collection and their interpretation. XC and SJ collaborated on bioinformatics analysis and statistical analysis. ZX co-designed experiments, discussed analyses, interpretation, presentation and wrote the paper. All authors read and approved the final manuscript.

\section{Ethics approval and consent to participate}

The current study was performed in accordance with the Declaration of Helsinki and approved by the Human Ethics Committee of Jinling Hospital. Written informed consent was obtained from each study participant.

\section{Consent for publication}

Not applicable.

\section{Competing interests}

The authors declare that they have no competing interests.

\section{References}

1. Zhou K, Zhang SS, Yan Y and Zhao S: Overexpression of transient receptor potential vanilloid 2 is associated with poor prognosis in patients with esophageal squamous cell carcinoma. Med Oncol 31: 17, 2014

2. Napier KJ, Scheerer M and Misra S: Esophageal cancer: A review of epidemiology, pathogenesis, staging workup and treatment modalities. World J Gastrointest Oncol 6: 112-120, 2014.

3. Siegel RL, Miller KD and Jemal A: Cancer statistics, 2015. CA Cancer J Clin 65: 5-29, 2015.

4. Toh Y, Egashira A and Yamamoto M: Epigenetic alterations and their clinical implications in esophageal squamous cell carcinoma. Gen Thorac Cardiovasc Surg 61: 262-269, 2013.

5. Song Y, Li L, Ou Y, Gao Z, Li E, Li X, Zhang W, Wang J, Xu L, Zhou Y, et al: Identification of genomic alterations in oesophageal squamous cell cancer. Nature 509: 91-95, 2014.

6. Ambros V: The functions of animal microRNAs. Nature 431: 350-355, 2004.

7. Lin S and Gregory RI: MicroRNA biogenesis pathways in cancer. Nat Rev Cancer 15: 321-333, 2015.

8. Gu J, Wang Y and $\mathrm{Wu} \mathrm{X}$ : MicroRNA in the pathogenesis and prognosis of esophageal cancer. Curr Pharm Des 19: 1292-1300, 2013.

9. Feber A, Xi L, Luketich JD, Pennathur A, Landreneau RJ, Wu M, Swanson SJ, Godfrey TE and Litle VR: MicroRNA expression profiles of esophageal cancer. J Thorac Cardiovasc Surg 135: 255-260, 2008

10. He B, Yin B, Wang B, Xia Z, Chen C and Tang J: MicroRNAs in esophageal cancer (Review). Mol Med Rep 6: 459-465, 2012.

11. Li P, Mao WM, Zheng ZG, Dong ZM and Ling ZQ: Down-regulation of PTEN expression modulated by dysregulated miR-21 contributes to the progression of esophageal cancer. Dig Dis Sci 58: 3483-3493, 2013.

12. Sugimura K, Miyata H, Tanaka K, Hamano R, Takahashi T, Kurokawa Y, Yamasaki M, Nakajima K, Takiguchi S, Mori M and Doki Y: Let-7 expression is a significant determinant of response to chemotherapy through the regulation of IL-6/STAT3 pathway in esophageal squamous cell carcinoma. Clin Cancer Res 18: 5144-5153, 2012.

13. Lin RJ, Xiao DW, Liao LD, Chen T, Xie ZF, Huang WZ, Wang WS, Jiang TF, Wu BL, Li EM and Xu LY: MiR-142-3p as a potential prognostic biomarker for esophageal squamous cell carcinoma. J Surg Oncol 105: 175-182, 2012.

14. Kano M, Seki N, Kikkawa N, Fujimura L, Hoshino I, Akutsu Y, Chiyomaru T, Enokida $\mathrm{H}$, Nakagawa $\mathrm{M}$ and Matsubara $\mathrm{H}$ : miR-145, miR-133a and miR-133b: Tumor-suppressive miRNAs target FSCN1 in esophageal squamous cell carcinoma. Int J Cancer 127: 2804-2814, 2010 .

15. Edge SB and Compton CC: The American joint committee on cancer: The 7th edition of the AJCC cancer staging manual and the future of TNM. Ann Surg Oncol 17: 1471-1474, 2010.

16. Livak KJ and Schmittgen TD: Analysis of relative gene expression data using real-time quantitative PCR and the 2(-Delta Delta C(T)) method. Methods 25: 402-408, 2001.

17. Clough $\mathrm{E}$ and Barrett $\mathrm{T}$ : The gene expression omnibus database. Methods Mol Biol 1418: 93-110, 2016.

18. Qian Y, Yuan J, Hu H, Yang Q, Li J, Zhang S, Jiang B, Shao C and Gong Y: The CUL4B/AKT/ $\beta$-catenin axis restricts the accumulation of myeloid-derived suppressor cells to prohibit the establishment of a tumor-permissive microenvironment. Cancer Res 75: 5070-5083, 2015.

19. Williams AH, Liu N, van Rooij E and Olson EN: MicroRNA control of muscle development and disease. Curr Opin Cell Biol 21: 461-469, 2009.

20. Kirby TJ and McCarthy JJ: MicroRNAs in skeletal muscle biology and exercise adaptation. Free Radic Biol Med 64: 95-105, 2013.

21. Zhao Y, Huang J, Zhang L, Qu Y, Li J, Yu B, Yan M, Yu Y, Liu B and Zhu Z: MiR-133b is frequently decreased in gastric cancer and its overexpression reduces the metastatic potential of gastric cancer cells. BMC Cancer 14: 34, 2014.

22. Zhao H, Li M, Li L, Yang X, Lan G and Zhang Y: MiR-133b is down-regulated in human osteosarcoma and inhibits osteosarcoma cells proliferation, migration and invasion, and promotes apoptosis. PLoS One 8: e83571, 2013.

23. Hu G, Chen D, Li X, Yang K, Wang H and Wu W: miR-133b regulates the MET proto-oncogene and inhibits the growth of colorectal cancer cells in vitro and in vivo. Cancer Biol Ther 10: 190-197, 2010. 
24. Higa LA, Wu M, Ye T, Kobayashi R, Sun $\mathrm{H}$ and Zhang $\mathrm{H}$ : CUL4-DDB1 ubiquitin ligase interacts with multiple WD40-repeat proteins and regulates histone methylation. Nat Cell Biol 8 : $1277-1283,2006$

25. Kerzendorfer C, Hart L, Colnaghi R, Carpenter G, Alcantara D, Outwin E, Carr AM and O'Driscoll M: CUL4B-deficiency in humans: Understanding the clinical consequences of impaired Cullin 4-RING E3 ubiquitin ligase function. Mech Ageing Dev 132: 366-373, 2011.

26. Dong J, Wang XQ, Yao JJ, Li G and Li XG: Decreased CUL4B expression inhibits malignant proliferation of glioma in vitro and in vivo. Eur Rev Med Pharmacol Sci 19: 1013-1021, 2015.

27. Chen Z, Shen BL, Fu QG, Wang F, Tang YX, Hou CL and Chen L: CUL4B promotes proliferation and inhibits apoptosis of human osteosarcoma cells. Oncol Rep 32: 2047-2053, 2014.

28. Jiang T, Tang HM, Wu ZH, Chen J, Lu S, Zhou CZ, Yan DW and Peng ZH: Cullin 4B is a novel prognostic marker that correlates with colon cancer progression and pathogenesis. Med Oncol 30: $534,2013$.

29. Jia S, Kobayashi R and Grewal SI: Ubiquitin ligase component $\mathrm{Cul} 4$ associates with $\mathrm{Clr} 4$ histone methyltransferase to assemble heterochromatin. Nat Cell Biol 7: 1007-1013, 2005.
30. Dumbliauskas E,LechnerE, Jaciubek M,Berr A,Pazhouhandeh M, Alioua M, Cognat V, Brukhin V, Koncz C, Grossniklaus U, et al: The Arabidopsis CUL4-DDB1 complex interacts with MSI1 and is required to maintain MEDEA parental imprinting. EMBO J 30: 731-743, 2011.

31. Higa LA, Yang X, Zheng J, Banks D, Wu M, Ghosh P, Sun H and Zhang H: Involvement of CUL4 ubiquitin E3 ligases in regulating CDK inhibitors Dacapo/p27Kip1 and cyclin E degradation. Cell Cycle 5: 71-77, 2006.

32. Nishitani H, Shiomi Y, Iida H, Michishita M, Takami T and Tsurimoto T: CDK inhibitor p21 is degraded by a proliferating cell nuclear antigen-coupled Cul4-DDB1Cdt2 pathway during S phase and after UV irradiation. J Biol Chem 283: 29045-29052, 2008.

This work is licensed under a Creative Commons Attribution-NonCommercial-NoDerivatives 4.0 International (CC BY-NC-ND 4.0) License. 